Cahiers Charlevoix

Études franco-ontariennes
Cahiers Charlevoix Études franco-ontariennes

or Crevenerix of

\title{
Phonétique et prosodie de l'emprunt intégral en franco-ontarien
}

\section{Ali Reguigui}

Volume 11, 2016

URI : https://id.erudit.org/iderudit/1039286ar

DOI : https://doi.org/10.7202/1039286ar

Aller au sommaire du numéro

\section{Éditeur(s)}

Société Charlevoix

Presses de l’Université d'Ottawa

ISSN

1203-4371 (imprimé)

2371-6878 (numérique)

Découvrir la revue

Citer cet article

Reguigui, A. (2016). Phonétique et prosodie de l'emprunt intégral en franco-ontarien. Cahiers Charlevoix, 11, 193-211.

https://doi.org/10.7202/1039286ar

\section{Résumé de l'article}

Le but de cette étude est de décrire et d'expliquer le traitement phonétique et prosodique de l'emprunt intégral fait par les Franco-Ontariens à l'anglais dans l'acte de parole. Il y a un nombre important de recherches portant sur la phonétique et la phonologie des emprunts intégrés et sur la manière dont les mots empruntés sont ajustés et reproduits selon les schèmes phonétiques de la langue receveuse. Toutefois, les études portant sur le traitement phonétique et prosodique de l'emprunt intégral en franco-ontarien font défaut. Cette étude se compose de quatre sections : dans la première, nous présenterons notre cadre théorique et un aperçu différentiel des systèmes phonétiques de l'anglais et du français. Nous exposerons ensuite notre problématique et avancerons quelques hypothèses. À la suite de cela, nous présenterons notre enquête et ses résultats et quelques perspectives de recherche. 


\title{
Phonétique et prosodie de l'emprunt intégral en franco-ontarien
}

\author{
Ali Reguigui \\ Département d'études françaises \\ Université Laurentienne
}




\section{SOMMAIRE}

$\begin{array}{ll}\text { INTRODUCTION } & 195\end{array}$

1. Contexte de l'étude et problématique de l'emprunt 196

2. Cadre théorique 198

3. Problématique et hypothèses 204

4. Méthodologie 205

5. Résultats (analyse et interprétation) 206

CONCLUSION / Discussion 209 


\section{Phonétique et prosodie de l'emprunt intégral en franco-ontarien}

\section{INTRODUCTION}

L'emprunt linguistique représente le procédé néologique fondé sur le recours aux ressources externes à la langue. Nous excluons de cette conception l'emprunt interne par lequel une langue opère des mouvements lexico-sémantiques entre la langue commune et les langues de spécialité (terminologisation) : par exemple : jupe (d'une voiture), bretelle (d'autoroute), souris (informatique). Dans toutes les langues, l'emprunt linguistique a plusieurs facettes. En effet, mis à part le calque (par exemple : tomber en amour de fall in love; bienvenu(e) de you are welcome, etc.) - qui est de l'ordre de la néologie sémantique par traduction et l'emprunt sémantique (par exemple : réaliser et faillir respectivement dans les sens anglais de to realise et to fail) -, l'emprunt intégré et l'emprunt intégral ont ceci de particulier qu'ils importent tous les deux de nouvelles formes lexicales en provenance directe ou indirecte d'une autre langue. Rappelons ici que l'emprunt intégré est le procédé par lequel une langue A emprunte un mot d'une langue $\mathrm{B}$ en l'adaptant à son phonétisme ou à sa morphosyntaxe (par exemple : bâdrer de to bother; bifteck de beefsteak), alors que l'emprunt intégral est le procédé par lequel une langue A emprunte un mot d'une langue $B$ sans adaptation phonétique ou morphosyntaxique (par exemple : hot dog, hamburger). L'emprunt compte ainsi parmi les procédés de création néologique dont dispose une langue pour enrichir son lexique. De ce point de vue, il est aussi légitime et naturel que la dérivation et la néologie sémantique ${ }^{1}$.

1. La dérivation est un procédé interne de création lexicale qui permet de créer de nouvelles unités lexicales par affixation, c'est-à-dire par « agglutination d'éléments lexicaux, dont un au moins n'est pas susceptible d'emploi indépendant, en 
Les langues empruntent plus fréquemment des unités du fonds lexical (noms, verbes, adjectifs) que des unités du répertoire grammatical (conjonctions, prépositions) et moins souvent des structures syntaxiques ou calques.

L'emprunt intégral, contrairement à l'emprunt intégré, a des conséquences phonétiques et phonologiques. Ces implications peuvent être plus ou moins grandes selon que les langues appartiennent ou non à la même famille de langues, partagent ou non un même système graphique.

La question de la phonétique et de la prosodie de l'emprunt intégral de manière générale, et particulièrement en francoontarien $^{2}$, nous intéresse à plus d'un égard. En effet, outre le désir d'explorer cet aspect encore vierge, nous nous intéressons à déterminer si l'emprunt intégral est vraiment intégral, car cela sous-entend une réalisation complète de l'emprunt aussi bien sur le plan phonétique que sur le plan prosodique. En outre, cette question est aussi au cœur des interrogations relatives au contact des langues et aux phénomènes qui en découlent : 1'alternance des codes, le transfert linguistique et l'assimilation.

\section{Contexte de l'étude et problématique de l'emprunt}

L'emprunt est un phénomène normal dans toutes les langues en

une forme unique ». (Jean Dubois et al., Dictionnaire de linguistique et des sciences du langage, Paris, Larousse, 1994). Quant à la néologie sémantique, elle est le procédé par lequel une langue crée de nouvelles unités de signification en utilisant des formes déjà existantes dans la langue en altérant leur structure sémique. La néologie sémantique utilise les procédés de la métaphore, de la métonymie, la restriction de sens, l'extension de sens, le jeu de synonymie et d'antonymie, etc. Ce procédé a pour conséquence la création d'homonymes. (Salah Mejri, « La reconnaissance automatique des néologismes de sens », dans Colloque des septièmes journées scientifiques Auf-Ltт, Bruxelles, 8 au 10 septembre 2005, s.p. http://perso.univ-lyon2.fr/ thoiron/ JS\%20LTT\%202005/pdf/MejriSalah.pdf (consulté le 19 juillet 2015) ; Jean-François Sablayrolles, La Néologie en français contemporain : examen du concept et analyse de productions néologiques récentes, Paris, Champion, 2000).

2. Le franco-ontarien est une variété du français laurentien parlée au Canada, notamment en Ontario et au Québec. Le français laurentien, dont les origines remontent au Québec et qui possède aussi des sous-variétés (entre autres le franco-ontarien et le franco-québécois qui font l'objet de variations régionales), s'oppose au français acadien qui possède lui aussi des sous-variétés qui sont parlées dans les provinces atlantiques, dans les zones limitrophes de ces provinces, de même que dans l'État du Maine et en Louisiane aux États-Unis. 
tant que procédé linguistique de néologie, mais il est davantage un comportement langagier dans les langues minoritaires en situation de contact. S'il est vrai que la langue minoritaire n'affecte pas le courant de la langue dans les situations majoritaires, c'est en situation minoritaire qu'elle fait montre de plus de dynamisme. Ce dynamisme est soutenu par la bilingualité de la communauté minoritaire ${ }^{3}$ et, donc, par sa capacité de passer d'une langue à l'autre dans une même interaction verbale. Ainsi, l'emprunt, dans ces situations, n'est pas nécessairement ou seulement dicté par le vide lexical dans la langue receveuse ou par les lacunes dans la compétence du locuteur ${ }^{4}$, mais aussi par le fait que le contact des langues dicte des modes de comportement langagier axés sur l'alternance des codes. Dans ces contextes, l'usage du vocable étranger est imposé par sa fréquence dans l'environnement langagier du locuteur, parfois même plus fréquemment que le vocable vernaculaire et, dans les situations majoritaires où règne la sécurité linguistique (mais aussi dans les situations minoritaires), l'emprunt peut être une mode qui reflète la force d'attrait qu'exercent une langue et une culture sur les locuteurs d'une autre langue et d'une autre culture ${ }^{5}$ ou un comportement marqueur de snobisme et de division de la société en classes sociolinguistiques ${ }^{6}$.

Les Franco-Ontariens constituent une communauté qui vit en situation de contact de langues, de minorité ou de bilinguisme forcé. Ils forment une communauté complexe dont les locuteurs présentent des caractéristiques socioéconomiques diverses et dont

3. Inga Vendelin et Sharo Peperkamp, « The Influence of orthography on loanword adaptations », Lingua, Nancy, Elsevier, vol. 116, 2006, p. 996-1007.

4. Simon Laflamme et Ali Reguigui, Deux Groupes linguistiques, une communication de masse, Paris, L'Harmattan, coll. " Logiques sociales », 1997 ; Simon Laflamme et Ali Reguigui, Homogénéité et distinction, Sudbury, Éditions Prise de parole, 2003.

5. Jean Pellerin, Pour l'amour de la langue française au Canada et au Québec, Montréal, Guérin, 1998 ; Uriel Weinreich, Languages in Contact, Paris, Mouton, 1974.

6. Chantal Bouchard, On n'emprunte qu'aux riches; la valeur sociolinguistique et symbolique des emprunts, Montréal, Fides, coll. " Grandes conférences », 1999. 
le bilinguisme se répartit sur un continuum allant de la dominance dans la première langue à la dominance dans la seconde langue, à une dominance équilibrée dans les deux langues, ou à un semilinguisme caractéristique d'un bilinguisme doublement soustractif. En outre, ils sont en contact physique avec la culture américaine. Pour toutes ces raisons, force est de constater qu'ils n'échappent pas à toutes conditions entourant l'occurrence de l'emprunt sous toutes ses formes.

\section{Cadre théorique}

L'emprunt, quoique procédé normal dans toutes les langues, n'a pas laissé de susciter des polémiques. Sur le plan diachronique, les débats sur l'emprunt ont été le reflet de tensions animant des sociétés divisées entre partisans du modernisme et partisans du purisme. Sur le plan synchronique, il y a une rupture entre le cadre législatif et normatif (académies, systèmes scolaires) et le cadre de l'usage, entre le politique et le social.

Sur le plan strictement linguistique, les études ont permis de faire la distinction entre l'emprunt en tant que procédé et l'emprunt en tant que résultat de ce procédé. En outre, il y a différentes notions relatives à l'emprunt. Certaines ont trait à la provenance de l'emprunt (anglicisme, gallicisme, germanisme, etc.), d'autres ont trait au type d'emprunt (emprunt de forme, emprunt de sens, calque, etc.) ${ }^{7}$. Enfin, certaines ont trait au moment de l'entrée et à la durée de l'emprunt (pérégrinisme, xénisme, etc. $)^{8}$.

7. Natalie Melanson, Choix linguistiques, alternances de langues et emprunts chez des Franco-Ontariens de Sudbury, Sudbury, Série monographique en sciences humaines/Institut franco-ontarien, 1996 ; Raymond Mougeon, "Les emprunts au vocabulaire de base de l'anglais en français ontarien ", dans Danielle Latin et Claude Poirier (dir.), Contacts de langues et identité culturelles : perspectives lexicographiques, Québec, Presses de l'Université Laval, 2000, p. 29-43 ; Valérie Raymond, «L'emprunt lexical à l'anglais dans le français oral des locuteurs bilingues de Sudbury (Ontario) : contrainte ou enrichissement pour une langue minoritaire ? », Mémoire de maîtrise, Québec, Université Laval, 2011.

8. Jean-Claude Boulanger, « Néologisme et terminologie », dans Office québécois de la langue française (éd.), Néologie en marche, série $B$ : langues de spécialité, Québec, Éditeur officiel du Québec, vol. 4, 1978, p. 5-127 ; Henri Bonnard, « L'Emprunt », Grand Larousse de la langue française, t. 2, Paris, Larousse, 1972, p. 1579-1590 ; Jean Darbelnet, « La norme lexicale et l'anglicisme au Québec », 
En outre, l'emprunt linguistique a été envisagé dans la perspective du rapport de force que les langues entretiennent les unes sur les autres, de l'attrait qu'elles exercent entre elles ou de l'apport culturel d'une langue à une autre langue ${ }^{9}$. L'emprunt a aussi été étudié sous l'aspect du comportement langagier de l'alternance de $\operatorname{codes}^{10}$.

En somme, l'emprunt est un phénomène très étudié sur les plans lexical et sociolinguistique, mais le traitement phonétique de l'emprunt n'a pas fait l'objet de plusieurs études et encore moins de traitement prosodique. L'intérêt porté au traitement phonétique de l'emprunt était surtout relié au rapport de celuici à l'orthographe et à la prononciation normalisée, des mots d'emprunts orthographiés ou adaptés graphiquement à la langue receveuse. Donc il s'agissait d'études d'ordre plutôt aménagemental que sociophonétique ${ }^{11}$. Nous ne reviendrons donc pas sur ces

dans Édith Bédard et Jacques Maurais (dir.), La Norme linguistique, Québec-Paris, Conseil de la langue française-Le Robert, 1983, p. 603-624; Louis Deroy, L'Emprunt linguistique, Paris, Les Belles Lettres, 1956 ; Louis Guilbert, La Créativité lexicale, Paris, Larousse, 1975 ; Louis Guilbert, " Aspects terminologique et linguistique du mot », Lebende Sprachen, Berlin-München, Langenscheidt, vol. 20, nº 6, 1975, p. 173-176 ; Alain Rey, Théories du signe et du sens, Paris, Éditions Klincksieck, 1976 ; « Néologisme : un pseudo-concept ? », Cahiers de lexicologie, Paris, DidierLarousse, vol. 28, n 1, 1976, p. 3-17 ; Guy Rondeau, Introduction à la terminologie, Chicoutimi, Gaétan Morin Éditeur, 1983 ; Raymond Mougeon, op. cit.

9. Leonard Bloomfield, Le Langage (traduit de l'anglais par J. Gazio, Language), Paris, Payot, [1933] 1970 ; Valérie Raymond, op. cit. ; Ali Reguigui, « Emprunt et normalisation en langue arabe », langue et linguistique, Québec, Presses de l'Université Laval, no 12, 1986, p. 197-227 ; Inga Vendelin et Sharo Peperkamp, « The Influence of orthography on loanword adaptations », Lingua, Nancy, Elsevier, vol. 116, 2006, p. 996-1007.

10. Melanson, op. cit. ; Shana Poplack, « The Propagation of loanwords within a speech community ", communication présentée au congrès NwAVE-XII, Montréal, Université de Montréal, 27-29 octobre 1983 ; Shana Poplack, "Contrasting patterns of code-switching in two communities ", dans H. J. Warkentyne (dir.), Papers from the International Conference on Methods in Dialectology, Victoria, C.-B., Université de Victoria, Département de linguistique, 1985, p. 363-385 ; Inga Vendelin et Sharo Peperkamp, op. cit. ; Moira Yip, « The Symbiosis between perception and grammar in loanword phonology », Lingua, Nancy, Elsevier, vol. 116, 2006, p. 950-975.

11. Marcel Danesi, Loanwords and Phonological Methodology, Didier, Ville LaSalle, 1985 ; Darlene LaCharite et Carole Paradis, " Phonological evidence for the bilingualism of borrowers ", Proceedings of the 2000 Annual Conference of the Canadian Linguistic Association, Ottawa, 2000, p. 221-232 ; id., " Category preservation and proximity versus phonetic approximation in loanword adaptation $»$. Linguistic Inquiry, Cambridge (MA), The Mit Press, vol. 36, 2005, p. 223-258 ; Diego Moran- 
nombreux aspects de l'emprunt qui ont été longuement étudiés sur les plans synchronique et diachronique de même que sur les plans linguistique et sociolinguistique.

Cependant, une étude ${ }^{12}$ portant sur la prosodie du francoontarien est venue compléter les études sur la phonétique du franco-ontarien ${ }^{13}$ et a traité des conséquences du contact des langues sur le franco-ontarien. Cette étude est d'une grande importance, car elle ouvre la porte à un domaine de recherche non encore exploré en franco-ontarien. Et même si son auteur n'a pas traité de la prosodie de l'emprunt et qu'il n'est pas arrivé à des conclusions probantes, cette étude mérite d'être poursuivie et approfondie.

Il appert donc que les lieux de carence en matière de recherche sur le franco-ontarien résident dans le domaine de la prosodie, plus particulièrement celui de la prosodie de l'emprunt. Ce constat va donc servir d'assise à notre point de départ dans cette recherche.

Ainsi, notre étude s'inscrit dans le cadre de la phonétique instrumentale, de la phonétique articulatoire et de la prosodie. Afin d'entamer l'étude, il est important de rendre compte de la différence entre le système phonétique français et le système phonétique anglais, ce qu'illustrent le tableau 1 et la figure 1.

dini, « The Phonology of Loan Words into Italian », (thèse de maîtrise), London, University College London, 2007 ; Office québécois de la langue française, Politique de l'emprunt linguistique, Québec, Publication du gouvernement du Québec, 2007 ; Carole Paradis et Jean-François Prunet, « Nasal vowels as two segments : evidence from borrowings », Language, Washington, Linguistic Society of America, vol. 76, 2000, p. 324-357 ; Ali Reguigui, op. cit.; Roman Retman, « L'Adaptation phonétique des emprunts à l'anglais en français ", La Linguistique, Paris, Les Presses universitaires de France, vol. 14, Fasc. 1, 1978, p. 111-124.

12. Jeff Tennant, « Rythme prosodique et contact des langues dans le français ontarien », dans France Martineau et Terry Nadasdi (dir.), Le Français en contact : hommage à Raymond Mougeon, Québec, Presses de l'Université Laval, 2011, p. 355-373.

13. Voir, entre autres, François Poiré, Stephanie Kelly et Darcie Williams, « La Réalisation des voyelles nasales en français de Windsor ", Revue Parole, Nancy, Université de Mons-Hainaut, vol. 39-40, 2006, p. 259-284 ; Pierre Léon, Phonétisme et prononciations du français, Paris, Nathan, 1992 ; Jeff Tennant, « Variation morphophonologique dans une langue en situation minoritaire : le français à North Bay ",, Revue du Nouvel-Ontario, Sudbury, Institut franco-ontarien, $n^{\circ} 20,1996, \mathrm{p} .1-136$; Alain Thomas, La Variation phonétique : cas du franco-ontarien, Montréal, Didier, coll. « Studia phonetica », n² 21, 1986. 
Tableau 1

Aperçu comparatif des consonnes en français et en anglais

\begin{tabular}{|c|c|c|c|c|c|c|c|c|c|}
\hline & & biblabiale & labiodentale & interdentale & Alvéolaire & palatale & vélaire & uvulaire & glottale \\
\hline \multirow{2}{*}{ occlusive } & Sourde & $\mathrm{p}_{3}$ & & & $t_{3}$ & & $\mathrm{k}_{3}$ & & \\
\hline & Sonore & $b_{3}$ & & & $\mathrm{~d}_{3}$ & & $g_{3}$ & & \\
\hline \multirow{2}{*}{ Nasale } & Sourde & & & & & & & & \\
\hline & Sonore & $\mathrm{m}_{3}$ & & & $\mathrm{n}_{3}$ & $\mathrm{n}_{1}$ & $\eta_{2}$ & & \\
\hline \multirow{2}{*}{ Fricative } & Sourde & & $f_{3}$ & $\theta_{2}$ & $\mathrm{~S}_{3}$ & $\int_{3} / t \int_{2}$ & & & \\
\hline & Sonore & & $v_{3}$ & $\partial_{2}$ & $\mathrm{Z}_{3}$ & $3_{1} / d_{3}$ & & $\mathrm{~B}_{1}$ & $\mathrm{~h}_{2}$ \\
\hline \multirow{2}{*}{ Latérale } & Sourde & & & & & & & & \\
\hline & Sonore & & & & $1_{3} / d_{2}$ & & & & \\
\hline \multirow{2}{*}{ glide } & Sourde & & & & & & & & \\
\hline & sonore & & & & $\mathrm{r}_{1} / \mathrm{I}_{2}$ & $\mathrm{u}_{1} / \mathrm{j}_{3}$ & $\mathrm{~W}_{3}$ & $\mathrm{R}_{1}$ & \\
\hline 1 Français & 2 Angla & & çais-anglais & & & & & & \\
\hline
\end{tabular}

Sur le plan consonantique, l'anglais et le français disposent d'un répertoire sensiblement le même. Toutefois, l'anglais se démarque du français par la présence de phonèmes oraux fricatifs interdentaux $/ \theta /$ (ex. : three, month) et / $/$ / (ex. : there, brother), de la glide alvéolaire rétroflexe/./ (ex. : court, burst), de la nasale vélaire /y/ (ex. : parking, skating) et de la fricative glottale sourde $/ \mathrm{h} /$ (ex. : $\underline{h o t}, \underline{h} a t)$. L'anglais possède également des phonèmes affriqués /t $\mathrm{f} /$ (ex. : chair, chat) et /dz/ (ex. : judge, jean, gin). L'anglais se démarque aussi par le phénomène d'aspiration consonantique en début de séquence phonétique (ex. : les mots pin, pot et pack sont respectivement prononcés avec une consonne initiale aspirée : $\left./ \mathrm{p}^{\mathrm{h}} /\right)$.

Le franco-ontarien, pour sa part, possède deux (ou même trois) variantes phonétiques du phonème /r/. Celui-ci peut se réaliser phonétiquement sous la forme d'une variante libre uvulaire voisée (communément appelée r grasseyé) [R], une variante libre alvéolaire voisée (aussi appelée r roulé) [r] et parfois même une variante contextuelle spirante rétroflexe voisée (aussi appelée $\mathrm{r}$ rétroflexe ou $\mathrm{r}$ anglais) $[\mathrm{x}]^{14}$. En outre, le franco-ontarien possède

14. Un locuteur a normalement, un /r/ soit uvulaire soit alvéolaire. Cependant, 
des affriqués $\left[\mathrm{t}_{\mathrm{s}}\right]$ et $\left[\mathrm{d}_{\mathrm{z}}\right.$ ] qui ont le statut phonologique de variantes combinatoires des phonèmes $/ \mathrm{t} / \mathrm{et} / \mathrm{d} /$. Ces variantes se manifestent devant les voyelles antérieures fermées $/ \mathrm{i} /, / \mathrm{y} /{ }^{15}$ : par exemple : le phonème $/ \mathrm{t} /$ dans le mot « poutine », sera prononcé $\left[\mathrm{t}_{\mathrm{s}}\right]>$ [put $\mathrm{s}$ ] $]$, le phonème /d/ dans le mot « dur » sera prononcé $\left[\mathrm{d}_{\mathrm{z}}\right]>\left[\mathrm{d}_{\mathrm{z}} \mathrm{YR}\right]$.

Figure 1

Aperçu comparatif des voyelles en français et en anglais

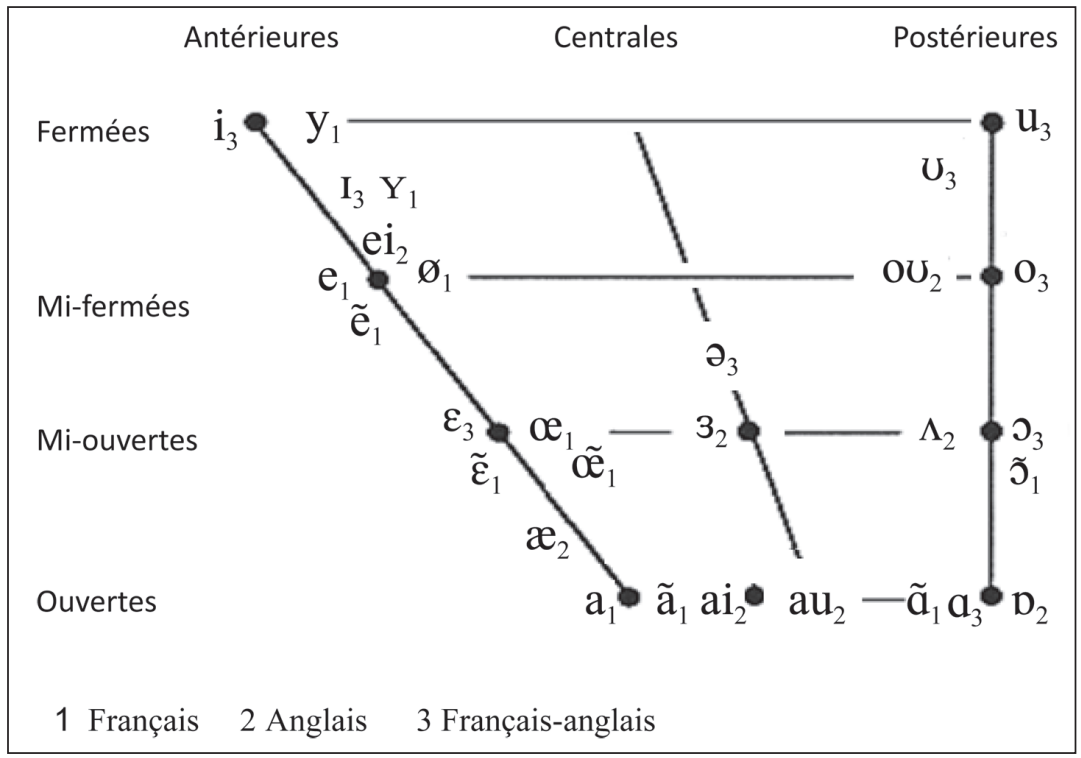

Sur le plan vocalique, il existe plusieurs différences entre le français et l'anglais. Il y a des différences au niveau des diphtongues.

nous remarquons parfois l'occurrence de ces deux variantes, en plus de la variante rétroflexe, dans le style articulatoire d'un même locuteur en fonction des registres et des situations de communication. En outre, il nous arrive d'observer l'occurrence des trois variantes dans le même tour de parole et même dans un même mot. Cette observation mérite que l'on mène des études supplémentaires pour déterminer les conditions d'occurrence d'allophones (variantes articulatoires) qui sont normalement en variation libre par rapport à ce qui pourrait s'annoncer aussi comme des variantes contextuelles en distribution complémentaire.

15. En français laurentien, les voyelles antérieures fermées /i/ et /y/ se réalisent tendues en syllabe ouverte ([i], [y]) et relâchées en syllabe fermée ([I], [Y]). 
Le français standard ne contient que des monophtongues, mais le français ontarien et le français laurentien de manière générale contiennent plusieurs diphtongues à titre de variantes libres des monophtongues. Le français ontarien et l'anglais ontarien ont des ressemblances à ce chapitre, mais ils entretiennent aussi des différences qualitatives. Pour ce qui est des voyelles simples ou monophtongues, il y a des différences systémiques comme on peut le voir dans la figure 1.

[ei] : Ang. : great, sleigh, engage, etc. / F.-o. : père, mère, etc.

[au]: Ang. : house, plow, gauge, etc. / F.-o. : pâtes, lâche, tasse, etc.

[ai] : Ang. : my, tide, thigh, buy, etc. / F.-o. : fête, bête, même, etc.

[0ซ] : Ang. : boat, coat, goat, etc. / F.-o. : côte, fausse, nôtre, etc.

Pour ce qui est des voyelles simples, le français dispose de deux séries de voyelles antérieures, une série arrondie et une série non arrondie, alors que l'anglais ne dispose que d'une série non arrondie. En outre, l'anglais ne dispose pas de voyelles nasales, alors que le français en a quatre standard et d'autres variantes régionales : surtout simples en franco-ontarien, simples et aussi diphtonguées en franco-québécois.

Sur le plan prosodique, le français et l'anglais se démarquent quant à l'accentuation. Alors que l'anglais dispose d'un système accentuel variable pouvant porter sur n'importe quelle syllabe du mot généralement sans aucune conséquence phonologique, le français dispose d'un accent fixe portant toujours sur la dernière syllabe du mot ou du groupe rythmique. En outre, alors que l'accent tonique anglais est culminatif (contenant de l'intensité), l'accent tonique français affecte plutôt la durée de la syllabe concernée, car, sur le plan de l'intensité, toutes les syllabes françaises se ressemblent (bien sûr, ici nous excluons l'accent expressif, qui n'a pas de valeur phonologique en français) ${ }^{16}$.

16. Jeff Tennant, ibid. 
Le français et l'anglais utilisent majoritairement des syllabes de structures $\mathrm{CV}, \mathrm{CVC}, \mathrm{CCV}$ et $\mathrm{VC}^{17}$, mais ils se distinguent quant à la fréquence de leur usage. Donc, de manière générale, la syllabe de type $\mathrm{CV}$, qui semble fréquente aussi bien en français qu'en anglais, est celle que préfère le français. Toutefois, l'anglais a une préférence plus marquée pour la syllabe de type $\mathrm{CVC}^{18}$.

Tableau 2

Aperçu comparatif des syllabes

\begin{tabular}{|l|c|c|}
\hline & Français & Anglais \\
\hline $\mathrm{CV}$ & $59,9 \%$ & $27,6 \%$ \\
\hline $\mathrm{CVC}$ & $17,1 \%$ & $31,8 \%$ \\
\hline $\mathrm{CCV}$ & $14,2 \%$ & $4,0 \%$ \\
\hline $\mathrm{VC}$ & $1,9 \%$ & $11,9 \%$ \\
\hline
\end{tabular}

\section{Problématique et hypothèses}

Le but de notre étude est d'explorer le sort phonétique réservé à l'emprunt intégral à l'anglais dans l'acte de parole des locuteurs franco-ontariens. Il s'agit pour nous de découvrir si le type de bilinguisme perçu par les jeunes Franco-Ontariens bilingues (francodominant, équilibré et anglo-dominant) reflète le phonétisme de l'emprunt intégral fait à l'anglais par ces locuteurs.

Nous partons des prémisses que la dominance en $\mathrm{L}_{1}$ appelle le maintien de tous les éléments du système (syntaxe, phonétique et prosodie), que l'équilibre entre langue première, $\mathrm{L}_{1}$, et langue seconde, $\mathrm{L}_{2}$, appelle l'accommodation (phonétique) de $\mathrm{L}_{2}$ et le maintien de $\mathrm{L}_{1}$ (syntaxe et prosodie), et que la dominance en $\mathrm{L}_{2}$ appelle le transfert à $\mathrm{L}_{2}$ (syntaxe, phonétique et prosodie). Autrement dit, nous considérons la syntaxe et la prosodie comme les noyaux durs du système. Ainsi, nous posons les hypothèses suivantes que nous articulons autour de deux axes : un axe phonétique et un axe prosodique.

17. $\mathrm{C}=$ consonne $; \mathrm{V}=$ voyelle. 1992.

18. Pierre Léon, Phonétisme et prononciations du français, Paris, Nathan, 
Hypothèses portant sur l'axe phonétique

1. Les franco-dominants suivront le modèle phonétique du français.

2. Les bilingues équilibrés suivront le modèle phonétique de l'anglais.

3. Les anglo-dominants suivront le modèle phonétique de l'anglais.

Hypothèses portant sur l'axe prosodique

1. Les franco-dominants suivront le modèle prosodique du français.

2. Les bilingues équilibrés suivront le modèle prosodique du français.

3. Les anglo-dominants suivront le modèle prosodique de l'anglais.

\section{Méthodologie}

\section{a) Sujets}

Par la méthode boule de neige, nous avons recruté 20 sujets adultes de langue maternelle française (13 femmes, 7 hommes), âgés entre 25 et 35 ans, ayant fait des études universitaires, d'origine ontarienne ou vivant en Ontario depuis au moins 15 ans, excepté pour un sujet du Québec qui vit en Ontario depuis 4 ans. Les sujets ont été classés selon leur auto-perception comme bilingues équilibrés (11), anglo-dominants (3), franco-dominants (6).

\section{b) Procédure}

Les sujets devaient mettre en phrase des emprunts intégraux courants en franco-ontarien à partir d'une liste préétablie (voir annexe). Ce faisant, nous avons enregistré les sujets en utilisant un appareil numérique.

\section{c) Traitement des données}

Les enregistrements sonores ont été ensuite transcrits en utilisant l'alphabet phonétique international (API), contrevérifiés par un accord interjuges et, ensuite, comparés à des enregistrements 
authentiquement anglais recueillis auprès d'un locuteur angloontarien $^{19}$.

En outre, un échantillon des enregistrements de chaque catégorie de sujets a été analysé avec la suite logicielle Computerized Speech Laboratory (CSL) et comparé à celui de l'anglais pour noter les différences acoustiques et prosodiques. Dans cette suite, nous avons utilisé la composante Real-Time Spectrogram, la composante Real-Time Pitch et la composante Analysis Synthesis Laboratory.

\section{Résultats (analyse et interprétation) \\ a) Analyse phonétique}

L'analyse des transcriptions phonétiques de tous les sujets n'a révélé que quelques rares différences portant surtout sur les phonèmes proches sur le plan articulatoire. Ces zones de variation consonantiques et vocaliques sont les glides alvéolaires $/ \mathrm{x} /$ contre $/ \mathrm{r} /$, les latérales $/ 4 /$ contre $/ / /$, la nasale vélaire $/ \mathrm{y} /$ contre la nasale et alvéolaire /n/, la voyelle quasi ouverte et non arrondies /æ/ contre la voyelle ouverte et arrondie /a/, la voyelle centrale semi-ouverte /3/ contre la voyelle centrale / /, la voyelle centrale / / contre la voyelle antérieure quasi fermée et relâchée /I/, de même que contre la voyelle postérieure orale / $/$ et la voyelle postérieure nasale /o/. Enfin, la voyelle postérieure ouverte et arrondie / $\mathrm{p} /$ contre la voyelle postérieure non arrondie / $/$ / . Certaines de ces variantes, en particulier les glides alvéolaires / $/ \mathrm{d} /$ contre / $/ \mathrm{r}$ ainsi que les latérales $/ 4 /$ contre $/ /$, sont en effet des variantes combinatoires en distribution complémentaire dans la langue anglaise. Donc, le fait qu'elles varient aussi dans la parole des Franco-Ontariens ne constitue pas un facteur significatif.

Hormis ces rares variations, tous les sujets, qu'ils soient bilingues équilibrés, franco-dominants ou anglo-dominants, ont prononcé les emprunts intégraux quasiment parfaitement selon

19. Nous tenons à remercier Valérie Raymond, doctorante en sciences humaines, qui a mené l'enquête auprès des sujets et a fait les enregistrements sonores, et Josée Mainguy, étudiante à la maîtrise en orthophonie, qui a fait la transcription phonétique du corpus recueilli. 
les modèles phonétiques anglais. Toutefois, à notre surprise, nous n'avons noté aucun phonème aspiré qui, normalement, serait caractéristique des productions des anglo-dominants.

\section{Tableau 3}

Aperçu des zones de variation consonantique

\begin{tabular}{|c|c|c|c|c|c|c|c|c|c|}
\hline & & biblabiale & labiodentale & interdentale & Alvéolaire & palatale & vélaire & uvulaire & glottale \\
\hline \multirow{2}{*}{ occlusive } & Sourde & $\mathrm{p}_{3}$ & & & $t_{3}$ & & $\mathrm{k}_{3}$ & & \\
\hline & Sonore & $b_{3}$ & & & $d_{3}$ & & $g_{3}$ & & \\
\hline \multirow{2}{*}{ Nasale } & Sourde & & & & & & & & \\
\hline & Sonore & $\mathrm{m}_{3}$ & & & $\mathrm{n}_{3}$ & $\mathrm{n}_{1}$ & $\eta_{2}$ & & \\
\hline \multirow{2}{*}{ Fricative } & Sourde & & $f_{3}$ & $\theta_{2}$ & $\mathrm{~S}_{3}$ & $\int_{3} / t \int_{2}$ & & & \\
\hline & Sonore & & $v_{3}$ & $\partial_{2}$ & $Z_{3}$ & $3_{1} / d_{3}$ & & $\mathrm{~b}_{1}$ & $\mathrm{~h}_{2}$ \\
\hline \multirow{2}{*}{ Latérale } & Sourde & & & & & & & & \\
\hline & Sonore & & & & $1_{3} / t_{2}$ & & & & \\
\hline \multirow{2}{*}{ glide } & Sourde & & & & & & & & \\
\hline & sonore & & & & $\mathrm{r}_{1} / \mathrm{I}_{2}$ & $\mathrm{U}_{1} / \mathrm{j}_{3}$ & $\mathrm{w}_{3}$ & $\mathrm{R}_{1}$ & \\
\hline
\end{tabular}

Figure 2

Aperçu des zones de variation vocalique

Antérieures

Centrales

Postérieures

Fermées

Mi-fermées
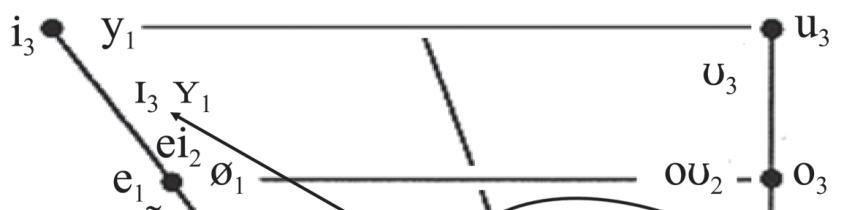

Mi-ouvertes

$\mathrm{e}_{1}$ $\tilde{\mathrm{e}}_{1}$

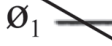

Ouvertes

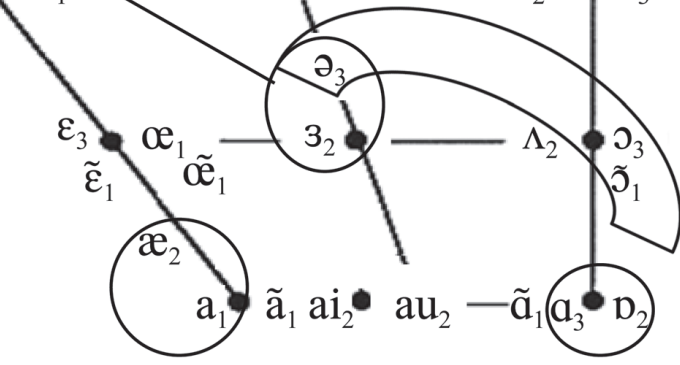

1 Français 2 Anglais 3 Français-anglais 


\section{b) Analyse prosodique}

Nous avons entrepris une analyse de l'accentuation des emprunts intégraux dans le corpus recueilli et l'avons comparé à l'accentuation de ces mêmes mots à partir des enregistrements faits en anglo-ontarien. Nous avons constaté un parfait accord entre les sujets, qu'ils soient bilingues équilibrés, franco-dominants ou anglo-dominants, tous ayant prononcé les emprunts intégraux d'après le modèle prosodique du français, c'est-à-dire en accentuant de manière systématique la syllabe finale. Comme nous l'avons indiqué plus tôt, l'accentuation d'après le modèle français s'effectue par une mise en valeur de la syllabe finale du mot ou du groupe rythmique qui se manifeste par une augmentation de la durée, ce qui est contraire à l'accentuation en anglais qui est variable et qui affecte l'intensité de la syllabe. Dans la figure suivante, les représentations accentuelles et spectrographiques de l'anglo-ontarien sont dans la colonne de gauche et celles du franco-ontarien sont dans la colonne de droite. La ligne supérieure indique la courbe mélodique, alors que la ligne inférieure indique la courbe accentuelle. L'on peut voir comment l'énergie accentuelle en franco-ontarien se trouve toujours en fin de segment, soit sur la dernière syllabe, alors qu'elle est variable en anglo-ontarien.

Au terme de ce parcours, rappelons d'abord nos hypothèses de départ :

\section{Hypothèses portant sur l'axe phonétique}

1. Les franco-dominants suivront le modèle phonétique du français.

2. Les bilingues équilibrés suivront le modèle phonétique de l'anglais.

3. Les anglo-dominants suivront le modèle phonétique de l'anglais.

Hypothèses portant sur l'axe prosodique

1. Les franco-dominants suivront le modèle prosodique du français

2. Les bilingues équilibrés suivront le modèle prosodique du français.

3. Les anglo-dominants suivront le modèle prosodique de l'anglais. 
Figure 3

Comparaison des représentations spectrographique et accentuelles de l'anglo-ontarien et du franco-ontarien

\section{Analyse accentuelle et spectrale}

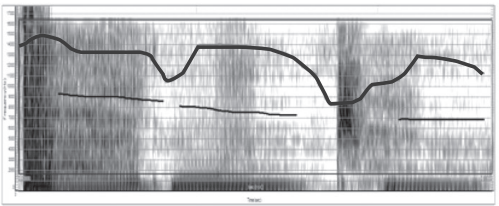

['bædmintən]

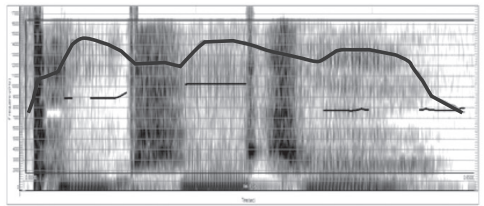

[kəm'pju:tə. $]$

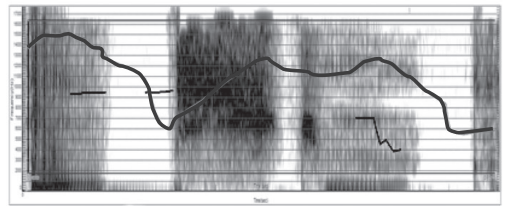

['łrpstık]

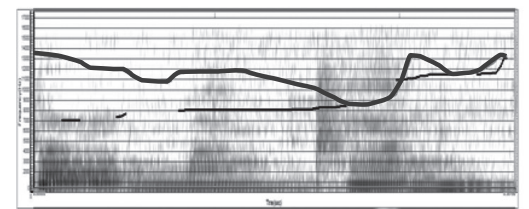

[bædmin'ti:n]

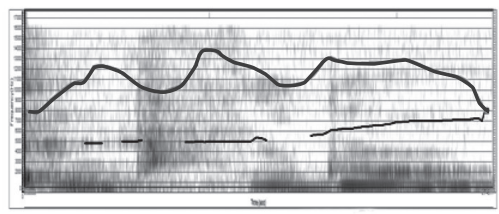

[kəmpju'tə:x]

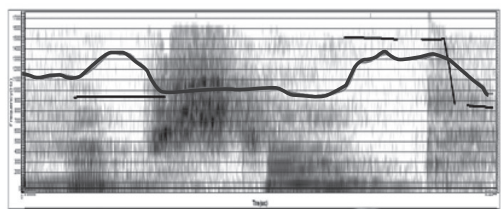

[tip'strik]

Pour les hypothèses phonétiques, notre première hypothèse celle selon laquelle les franco-dominants suivraient le modèle phonétique du français - est infirmée, alors que la deuxième et la troisième sont confirmées : les bilingues équilibrés et les anglodominants suivent le modèle phonétique de l'anglais.

Pour ce qui est de l'analyse prosodique, force est de constater que tous les sujets - les franco-dominants, les bilingues équilibrés ainsi que les anglo-dominants - ont manifesté un comportement prosodique conforme au système de la langue française. Ainsi, nos deux premières hypothèses sont confirmées, alors que la troisième est infirmée.

\section{Conclusion / Discussion}

Les résultats que nous venons d'obtenir sont importants à plusieurs égards. En effet, en Ontario français, on a fait un grand nombre 
d'études, entre autres, sur l'état de la langue des Franco-Ontariens, de leur compétence, de leur bilinguisme et de leur comportement langagier. Plusieurs théories opposées ont été élaborées. Certaines rassurantes et d'autres alarmistes, mais les critères d'analyse ne font pas l'unanimité. Il y a cependant un consensus à l'effet que le noyau dur de la langue est la morphosyntaxe, car il est rare que cette composante soit affectée par des emprunts à d'autres langues. Les domaines du vocabulaire et de la phonétique sont, par contre, plus permissifs et perméables. Mais il y a un autre domaine qui, suite à cette étude, s'avère un noyau dur de la langue, celui de la prosodie. En effet, la prosodie est une composante caractéristique du système linguistique et résiste de ce fait au transfert. D'ailleurs, il y a des instances dans les études sur les alternances de codes où les productions sont tellement enchevêtrées que le chercheur ne parvient pas à décider si la langue de base dans l'alternance de codes est $\mathrm{L}_{1}$ ou $\mathrm{L}_{2}$. L'analyse prosodique pourrait devenir un facteur déterminant dans la levée de l'ambigüité.

Nous ne pouvons pas clore notre article sans en indiquer les limites. D'abord, nous pensons que le nombre de sujets ne permet pas une bonne compréhension du phénomène. Nous aurons besoin d'augmenter l'échantillon et de couvrir une plus grande variété de sujets afin d'atteindre une représentativité sociale. En outre, la catégorisation selon le type de bilinguisme est en théorie juste, mais il nous faudra nous éloigner de l'auto-évaluation et nous fonder sur des critères formels et objectifs pour déterminer cet état. 


\section{Annexe}

Liste des emprunts intégraux utilisés dans cette étude

\begin{tabular}{|c|c|}
\hline backpack & $\log$ \\
\hline badminton & magazine \\
\hline basketball & marketing \\
\hline best-seller & meatloaf \\
\hline bib & minivan \\
\hline blog & mouse \\
\hline break & muffin \\
\hline brownie & mug \\
\hline business & muscle shirt \\
\hline carry-on & pancake \\
\hline cart (chariot) & parking \\
\hline chairlift & plug \\
\hline cheese ball & pop \\
\hline computer & poster \\
\hline country & printer \\
\hline cream cheese & purse \\
\hline crib & quiz \\
\hline crockpot & running shoe \\
\hline cupcake & sandwich \\
\hline ditch & screen \\
\hline drink & shift \\
\hline football & shopping \\
\hline french toast & size \\
\hline gang & slogan \\
\hline gravy & snowshoe \\
\hline hairstyle & soccer \\
\hline headband & story ou success story \\
\hline high heel & suitcase \\
\hline jazz & sundae \\
\hline job & sweatshirt \\
\hline jogging & switch \\
\hline joke & tennis \\
\hline keyboard & toboggan \\
\hline laptop & $t$-shirt \\
\hline lipstick & voicemail \\
\hline
\end{tabular}

